\title{
Characterising the Dwarf Nova Population of the Catalina Real-time Transient Survey
}

\author{
Deanne de Budè ${ }^{1}$, Patrick Woudt ${ }^{1}$ and Brian Warner ${ }^{1,2}$ \\ ${ }^{1}$ Department of Astronomy, University of Cape Town, Cape Town, South Africa \\ ${ }^{2}$ School of Physics and Astronomy, Southampton University, Southampton, UK \\ email: deanne@ast.uct.ac.za
}

\begin{abstract}
Results of a high-speed photometric study of dwarf novæ in the Catalina Real-time Transient Survey are given. A population of faint dwarf novæ near the orbital period minimum is detected. At the shortest periods there is a correlation between orbital period and outburst interval.
\end{abstract}

Keywords. dwarf novæ, Catalina Real-time Transient Survey, orbital period minimum

Over the past decade the Astronomy Department at the University of Cape Town has carried out high-speed optical photometry of faint southern-hemisphere cataclysmic variables (Woudt \& Warner 2010), using candidates taken largely from the Sloan Digital Sky Survey (SDSS). From late 2009 the survey has used CVs from the Catalina Real-time Transient Survey (CRTS: Drake et al. 2009), which extends to fainter magnitudes than the SDSS and identifies objects that vary by more than $2 \mathrm{mag}$ in their (approximately) 7-year light curves.

We have observed 56 of these stars, most of which are newly recognised CVs, using the 40-in and 74-in reflectors at the Sutherland site of the South African Astronomical Observatory, equipped with the UCT CCD photometer (O'Donoghue 1995). This has led to the measurement of orbital periods $\left(\mathrm{P}_{\text {orb }}\right)$ for 19 systems with magnitudes $\lesssim 20.5$, almost all of which are near the minimum orbital period, i.e., 80-90 mins. This is in line with the result obtained by (Gänsicke et al. 2009), based on SDSS systems, that the high space density of short period CVs predicted from population synthesis models can be found as faint CVs. Supplementing our $\mathrm{P}_{\text {orb }} \mathrm{s}$ with others for CRTS CVs available in the literature, we find the distributions shown in Fig. 1

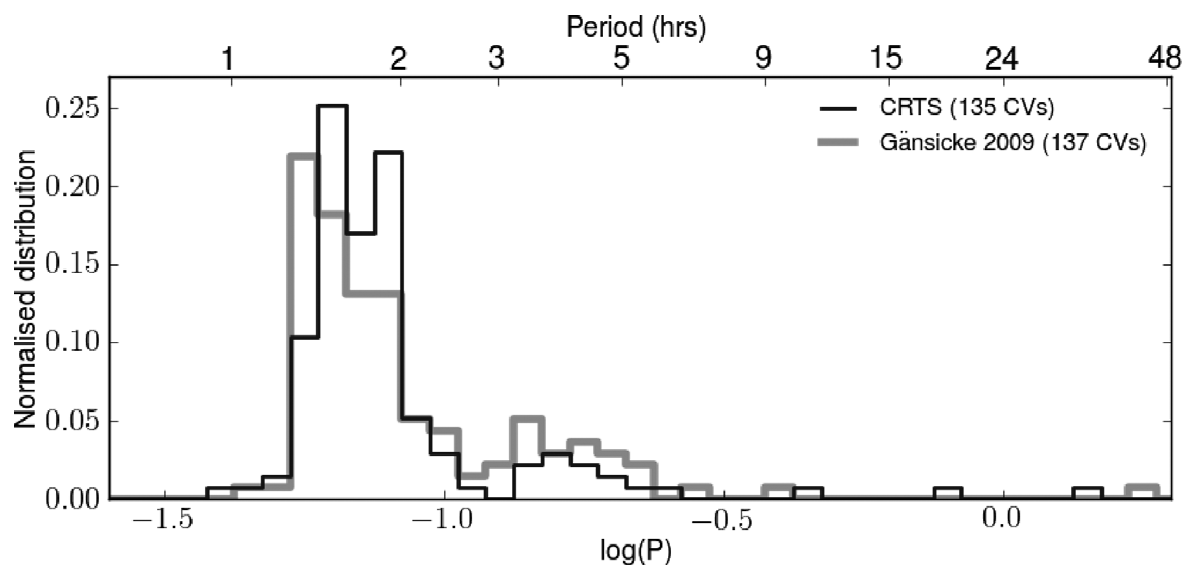

Figure 1. $\mathrm{P}_{\text {orb }}$ distributions from the SDSS (Gänsicke et al. 2009) and CRTS surveys. 


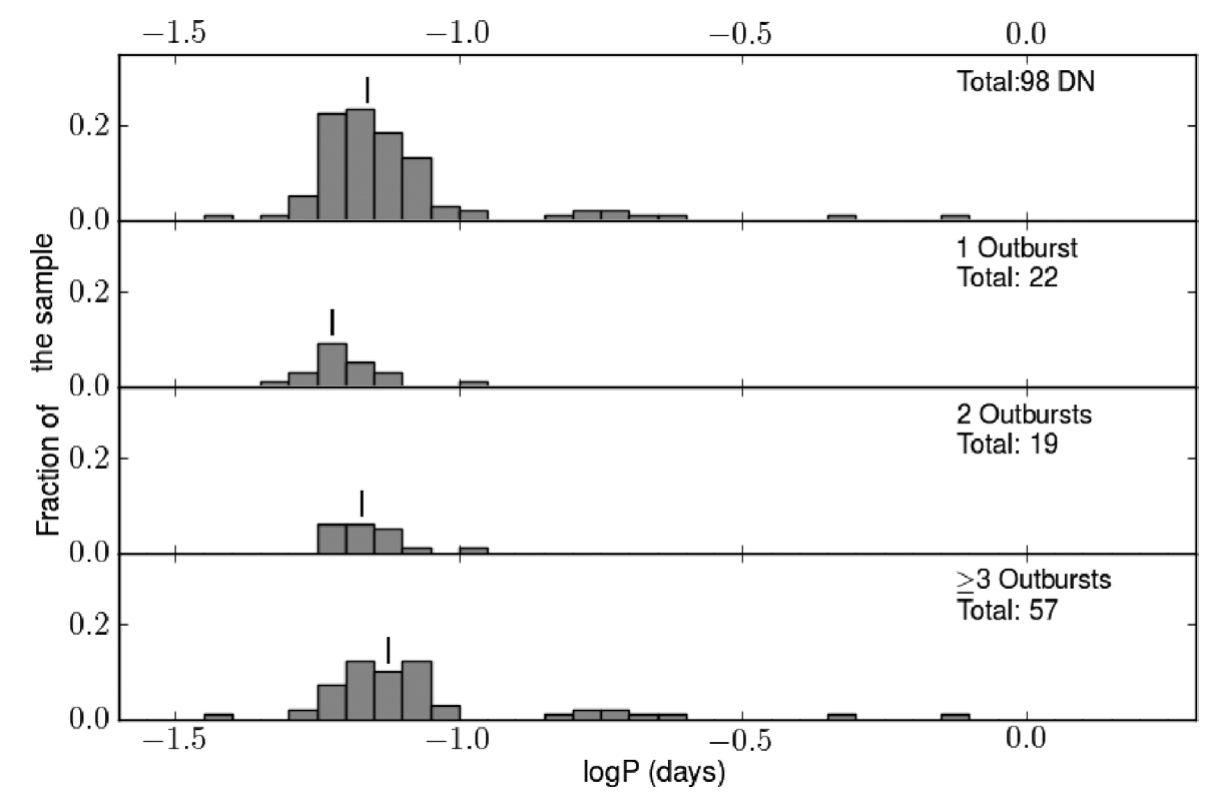

Figure 2. $\mathrm{P}_{\text {orb }}$ distributions of the CRTS stars according to outburst class. The vertical bars indicate the median values for each class and are given respectively by $1.66 \mathrm{~h}, 1.43 \mathrm{~h}, 1.62 \mathrm{~h}$ and $1.80 \mathrm{~h}$.

Evolutionary models also predict that most CVs should have evolved past the minimum $\mathrm{P}_{\text {orb }}$ back towards longer values (post-bounce $\mathrm{CVs}$ ), with lower mass transfer rates and rarer dwarf nova outbursts. We have classified the CRTS long-term light curves according to whether one, two, or more outbursts were detected; the $\mathrm{P}_{\text {orb }}$ histograms for the three types are shown in Fig. 2.

There is a clear correlation with $\mathrm{P}_{\text {orb }}$ in the sense that the least frequent outbursts occur among the dwarf novæ at the shortest orbital periods. Some of these may be post-bounce CVs.

\section{Acknowledgements}

DdB is funded by the South African Square Kilometre Array Project and the University of Cape Town, PW and BW's research is supported by the National Research Foundation and by the University of Cape Town.

\section{References}

Drake, A., et al. 2009, ApJ, 696, 870.

Gänsicke, B. T., et al. 2009, MNRAS, 397, 2170.

O'Donoghue, D. 1995, Balt. Astr., 4, 517.

Woudt, P. A. \& Warner, B. 2010, MNRAS, 403, p. 398 